\title{
Golden rule decay versus Lyapunov decay of the quantum Loschmidt echo
}

\author{
Ph Jacquod, ${ }^{1}$ P G Silvestiov, ${ }^{12}$ and C W J Beenakker ${ }^{1}$ \\ ${ }^{1}$ Instituut Lorentz Unvversitelt Leiden PO Box 95062300 RA Leiden The Netherlands \\ ${ }^{2}$ Budker Institute of Nuclear Physics 630090 Novosibirsk Russia \\ (Received 19 July 2001 published 15 October 2001)
}

\begin{abstract}
The overlap of two wave packets evolving in time with slightly different Hamiltontans decays exponentially $\propto e^{-\gamma t}$, for perturbation strengths $U$ greater than the level spacing $\Delta$ We present numerical evidence for a dynamical system that the decay rate $\gamma$ is given by the smallest of the Lyapunov exponent $\lambda$ of the classical chaotic dynamics and the level broadening $U^{2} / \Delta$ that follows from the golden rule of quantum mechanics This implies the range of validity $U>\sqrt{\lambda \Delta}$ for the perturbation-strength independent decay rate discovered by Jalabert and Pastawskı [Phys Rev Lett 86, 2490 (2001)]
\end{abstract}

DOI 10 1103/PhysRevE 64055203

PACS number(s) $0545 \mathrm{Mt}, 0545 \mathrm{Pq}, 4250 \mathrm{Md}, 7660 \mathrm{Lz}$

The search for classical Lyapunov exponents in quantum mechanics is a celebrated problem in quantum chaos [1] Motivated by NMR experiments on spin echoes [2], Jalabert and Pastawsk1 [3] have given analytical evidence, supported by computer simulations [4], that the Lyapunov exponent governs the time dependence of the fidelity

$$
M(t)=\left|\left\langle\psi\left|\exp (\imath H t) \exp \left(-{ }_{\imath} H_{0} t\right)\right| \psi\right\rangle\right|^{2},
$$

with which a wave packet $\psi$ can be reconstructed by inverting the dynamics with a perturbed Hamiltonian $H=H_{0}$ $+H_{1}$ They have called this the problem of the "quantum Loschmidt echo" The fidelity $M(t)$ can equivalently be interpreted as the decaying overlap of two wave functions that start out identically and evolve under the action of two slightly different Hamıltonıans, a problem first studied in perturbation theory by Peies [5]

Perturbation theory breaks down once a typical matrix element $U$ of $H_{1}$ connectıng different eigenstates of $H_{0}$ becomes greater than the level spacing $\Delta$ Then the eigenstates of $H$, decomposed into the eigenstates of $H_{0}$, contain a large number of non-negligible components The distribution $\rho(E)$ (local spectral density) of these components over energy has a Lorentzian form

$$
\rho(E)=\frac{\Gamma}{2 \pi\left(E^{2}+\Gamma^{2} / 4\right)},
$$

with a spreading width $\Gamma \simeq U^{2} / \Delta$ given by the golden rule $[6,7]$ A simple calculation in a 1andom-matix model gives an average decay $\bar{M} \propto \exp (-\Gamma t)$ governed by the same golden iule width This should be contrasted with the exponentral decay $\bar{M} \propto \exp (-\lambda t)$ obtained by Jalabert and Pastawski [3], which is governed by the Lyapunov exponent $\lambda$ of the classical chaotic dynamics

Since the random-matix model has by construction an infinite Lyapunov exponent, one way to unity both results would be to have an exponentral decay with a 1 ate set by the smallest of $\Gamma$ and $\lambda$ We will in what follows present nu merical evidence for this scenar1o, using a dynamical system in which we can vaty the relative magnitude of $\Gamma$ and $\lambda$ There exists a third energy scale, the inverse of the Ehi enfest time $\tau_{E}$, that is smaller than the Lyapunov exponent by a factor logarithmic in the system's effective Planck constant In our numerics we do not have enough orders of magnitude between $1 / \tau_{\mathrm{E}}$ and $\lambda$ to distınguish between the two, so that our findings iemain somewhat inconclusive in this respect

Because $\Gamma$ cannot become bigger than the band width $B$ of $H_{0}$ (we are interested in the regime $H_{1}<H_{0}$ ), a consequence of a decay $\bar{M} \propto \exp [-t \min (\lambda, \Gamma)]$ is that the regime of Lyapunov decay can only be reached with increasing $U$ if $\lambda$ is considerably less than $B$ That would exclude typical fully chaotic systems, in which $\lambda$ and $B$ are comparable, and set limits of observability of the Lyapunov decay

The crossover from the golden rule regime to a regime with a perturbation-strength independent decay, obtained here for the Loschmidt echo, should be distinguished from the conesponding crossover in the local spectial density $\rho(E)$, obtained by Cohen and Heller [8] The Fourier transform of $M(t)$ would be equal to $\rho(E)$ if $\psi$ would be an eigenstate of $H_{0}$ rather than a wave packet The choice of a wave packet instead of an eigenstate does not matter in the golden rule regime, but is essential for a decay rate given by the Lyapunov exponent

The dynamical model that we have studied is the kicked top [9], with Hamıltonian

$$
H_{0}=(\pi / 2 \tau) S_{3}+(K / 2 S) S_{z}^{2} \sum_{n} \delta(t-n \tau)
$$

It describes a vector spin (magnitude $S$ ) that undergoes a free piecession around the $y$ axis perturbed periodically (period $\tau)$ by sudden totations around the $z$ axis over an angle propoitional to $S_{z}$ The time evolution of a state after $n$ periods is given by the $n$th power of the Floquet operator

$$
F_{0}=\exp \left[-{ }_{l}(K / 2 S) S_{z}^{2}\right] \exp \left[-{ }_{l}(\pi / 2) S_{j}\right]
$$

Depending on the kicking strength $K$, the classical dynamics is regula1, pattially chaotic, or fully chaotic The dependence of the Lyapunov exponent $\lambda$ on $K$ is plotted in the inset to Fig 1 (cf Ref [10]) The error bats reflect the spread in $\lambda$ in different regrons of phase space, in particular the presence of sslands of stability For $K \geqslant 9$ the enor bars vanish because the system has become fully chaotic For the reversed time 


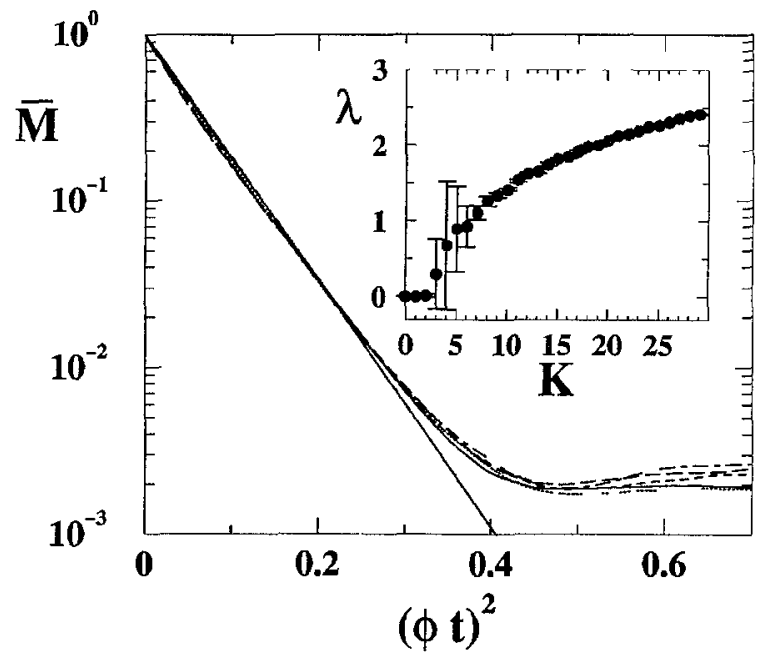

FIG 1 Decay of the average fidelity $\bar{M}$ for the quantum kicked top with $K=131$ and $S=500$, as a function of the squared rescaled time $(\phi t)^{2}$ The perturbation strengths range between $\phi=10^{-7}$ and $10^{-6}$ The straight line corresponds to the Gaussian decay (6) valıd in the perturbative regime Inset Numerically computed Lyapunov exponent for the classical kicked top as a function of the kicking strength $K$ Dots correspond to averages taken over $10^{4}$ initial conditions (see Ref [10]) The error bars reflect different results obtaned with different initial conditions The vanishing of error bars indicates the disappearance of islands of regular dynamics

evolution we introduce as a perturbation a periodic rotation of constant angle around the $x$ axis, slightly delayed with respect to the kicks $H_{0}$,

$$
H_{1}=\phi S_{x} \sum_{n} \delta(t-n \tau-\epsilon)
$$

The conesponding Floquet operator is $F=\exp \left(-\iota \phi S_{x}\right) F_{0}$ We have set $\hbar=1$ and in what follows we will also set $\tau$ $=1$ for ease of notation

Both $H$ and $H_{0}$ conserve the spin magnitude We choose the initial wave packets as coherent states of the spin SU(2) group [11], 1 e, states that mınımize the Heisenberg uncertainty in phase space (in our case on a sphere of fixed radius) at the effective Planck constant $h_{\mathrm{eff}} \sim S^{-1}$ The conesponding Ehrenfest time is $\tau_{\mathrm{E}}=\lambda^{-1} \ln S$ [12] We take $S=500$ and average $M(t=n)=\left|\left\langle\psi\left|\left(F^{\dagger}\right)^{n} F_{0}^{n}\right| \psi\right\rangle\right|^{2}$ over 100 mitial cohe1ent states $\psi$

We first show results in the fully chaotic regime $K>9$, where we choose the initial states randomly over the entire phase space The local spectial density $\rho(\alpha)$ of the eigenstates of $F$ (in the basis of the eigenstates of $F_{0}$ with eigenphases $\alpha$ ) is plotted for three different $\phi$ 's in the inset to Fig 2 The curves can be fitted by Lorentzians fiom which we extract the spreading width $\Gamma$ (It is given up to numerical coefficients by $\Gamma \simeq U^{2} / \Delta, U \simeq \phi \sqrt{S}, \Delta \simeq 1 / S$ ) The golden rule regime $\Gamma \geqslant \Delta$ is entered at $\phi_{c} \approx 17 \times 10^{-4}$ Fo1 $\phi \ll \phi$ c we are in the perturbative regime, where eigenstates of $F$ do not appieciably differ fiom those of $F_{0}$ and eigenphase dif-

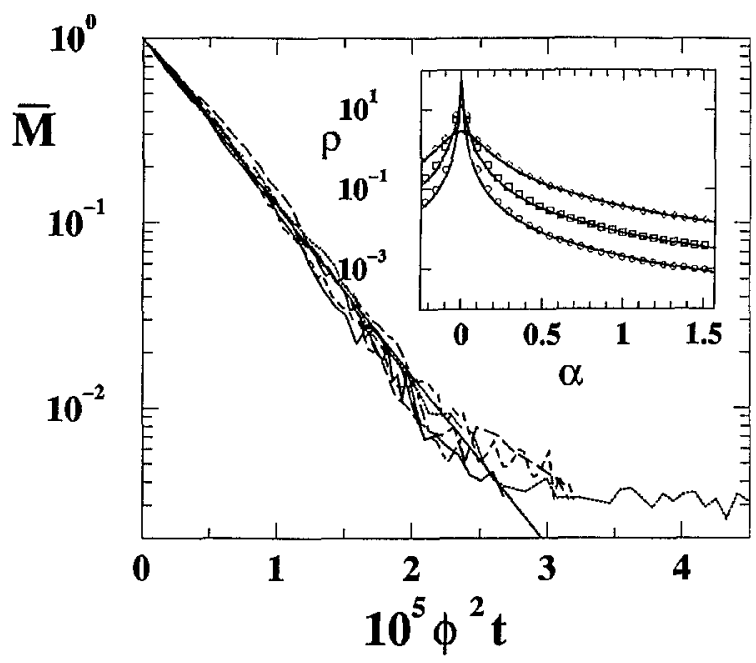

FIG 2 Decay of $\bar{M}$ in the golden rule regime for kicking strengths $K=131,171$, and 211 as a function of the rescaled time $\phi^{2} t$ Perturbation strengths range from $\phi=10^{-4}$ to $10^{-3}$ Inset Local spectral density of states for $K=131$ and perturbation strengths $\phi=25 \times 10^{-4}, 5 \times 10^{4}, 10^{-3}$ The sold curves are Lorentzian fits, from which the decay rate $\Gamma \approx 084 \phi^{2} S^{2} 1$ s extracted The solid line in the main plot gives the decay $\bar{M}$ $\propto \exp (-\Gamma t)$ with this value of $\Gamma$

ferences can be calculated in first-order peiturbation theory We then expect the Gaussian decay

$$
\bar{M} \propto \exp \left(-U^{2} t^{2}\right) \Rightarrow \ln \bar{M} \propto(\phi t)^{2}
$$

This decay is evident in Fig 1, which shows $\bar{M}$ as a function of $(\phi t)^{2}$ on a semilogarithmic scale for $\phi \leqslant 10^{-6}$ The decay (6) stops when $\bar{M}$ approaches $M_{\infty}=1 / 2 S$, being the inverse of the dimension of the Hilbert space This saturation ieflects the finiteness of the system and eventually prevalls at long times independently of the strength of the perturbation

For $\phi>\phi_{c}$ one enters the golden rule regime, where the Lorentzian spreading of eigenstates of $F$ over those of $F_{0}$ results in the exponential decay

$$
\bar{M} \propto \exp \left(-U^{2} t / \Delta\right) \Rightarrow \ln \bar{M} \propto \phi^{2} t
$$

The data presented in Fig 2 clearly confirm the validity of the scaling (7) There is no dependence of $\bar{M}$ on $K$ in this regime of moderate (but nonperturbative) values of $\phi, 1 \mathrm{e}$, no dependence on the Lyapunov exponent ( $\lambda$ varies by a factor of 14 for the different values of $K$ in Fig 2)

We cannot satısfy $\lambda<\Gamma$ in the fully chaotic regime, for the 1eason mentioned in the Introduction The band width $B$ (which is an upper limit for $\Gamma$ ) is $B=\pi / 2$ (in units of $1 / \tau$ ), while $\lambda \geqslant 1$ for fully developed chaos in the kicked top (see the inset to Fig 1) For this reason, when the perturbation strength $\phi$ is further increased, the golden rule decay rate saturates at the bandwidth - before reaching the Lyapunov exponent This is shown in Fig 3 There is no tiace of a Lyapunov decay in this fully chaotic regime 


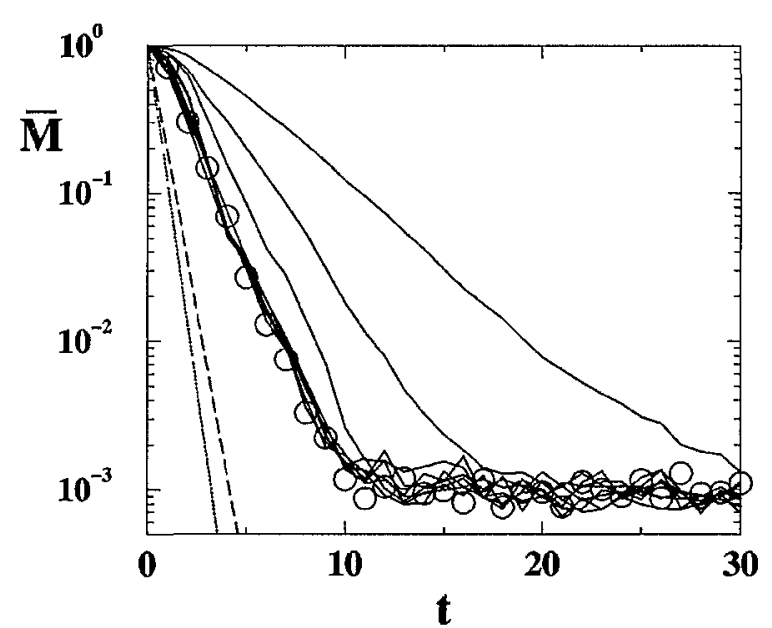

FIG 3 Decay of $\bar{M}$ in the golden rule regime without rescaling of time, for $K=131, \phi=j \times 10^{3}, \quad(J=1,15,2, \quad 5)$ (solid curves) and $K=211, \phi=3 \times 10^{-3}$ (circles) Dashed and dotted lines show exponential decays with Lyapunov exponents $\lambda=165$ and 212 , corresponding to $K=131$ and 211 , respectively The decay slope saturates at $\phi \approx 25 \times 10^{-3}$, when $\Gamma$ reaches the bandwidth

We therefore reduce $K$ to values in the range $27 \leqslant K$ $\leqslant 42$, which allows us to vary the Lyapunov exponent ove1 a wider range between 022 and 072 In this range the classical phase space is mixed and we have coexisting regula1 and chaotic trajectories We choose the initial coherent states in the chaotic region (identified numerically through the paiticipation ratio) Because the chaotic region still occupies more than $80 \%$ of the phase space for the smallest value of $K$ considered, nonuniveisal effects (e $g$, nonzeio overlap of our initial wavepackets with regular eigenfunctions of $F_{0}$ or $F$ ) should be negligible We expect a crossover from the golden rule decay (7) to the Lyapunov decay [3]

$$
\bar{M} \simeq \exp (-\lambda t) \Rightarrow \ln \bar{M} \propto \lambda t,
$$

once $\Gamma$ exceeds $\lambda$ This expectation is botne out by our numerical simulations, see Fig 4

In conclusion, we have presented numerical evidence for the existence of thiee distinct regimes of exponential decay of the Loschmidt echo the peiturbative regime (6), the golden rule 1egime (7), and the Lyapunov regime (8) The

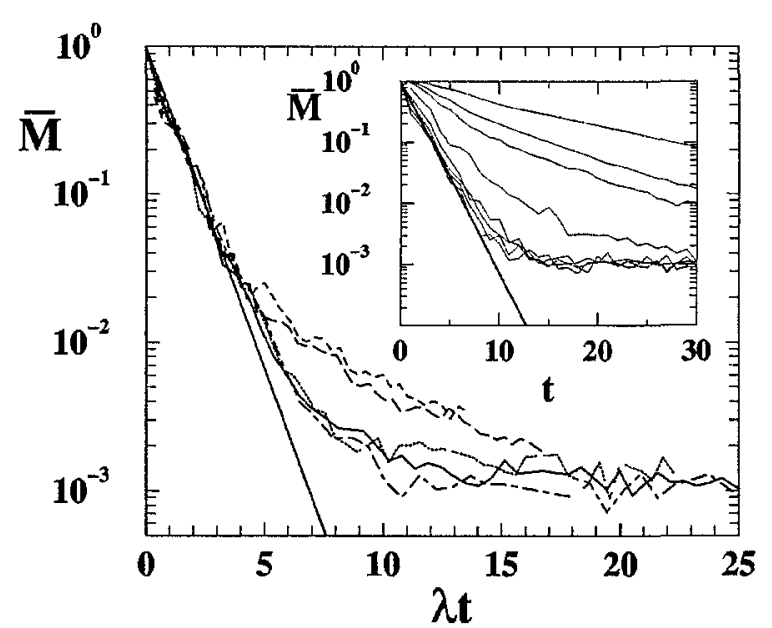

FIG 4 Decay of $\bar{M}$ in the Lyapunov regime, for $\phi$ $=21 \times 10^{-3}, K=27,33,36,39,42$ The time is rescaled with the Lyapunov exponent $\lambda$, ranging from $022-072$ The straight solıd line indicates the decay $\bar{M} \propto \exp (-\lambda t)$ Inset $\bar{M}$ for $K=42$ and different $\phi={ }_{J} \times 10^{4}, J=1,2,3,4,5,9,17,25$ The decay slope saturates at the value $\phi \approx 17 \times 10^{-3}$ for which $\Gamma \approx \lambda$, even though $\Gamma$ keeps on increasing This demonstrates the decay law $\bar{M}$ $\propto \exp (-\gamma t)$ with $\gamma=\min (\Gamma, \lambda)$

peiturbation strength independent decay in the Lyapunov regime is reached in our simulation if $\lambda<\Gamma$, which pievents its occurrence for fully developed chaos in the model consideied here Our numeitcs are limited by a relatively small window between $\lambda$ and $1 / \tau_{\mathrm{E}}$ (a factor $\ln S \approx 6$ ) It 1emains to be seen if the Lyapunov decay can be observed under conditions of fully developed chaos and $\Gamma<\lambda$ by increasing $S$ so that $1 / \tau_{\mathrm{E}}$ becomes larger than $\Gamma$ It is noteworthy that for a Lyapunov decay $\bar{M} \propto \exp (-\lambda t)$, the saturated fidelity $M_{\infty}$ $=1 / 2 S$ is reached at the Ehrenfest time $\tau_{\mathrm{E}}$ (as can also be seen $m$ Fig 4), so that a Lyapunov decay for $t \leqslant \tau_{E}$ rules out golden rule decay for later times Similar investigations in strongly chaotic systems with small Lyapunov exponents (like the Bunimovich stadium with short straight segments) are highly desirable

Th1s work was supported by the Swiss National Science Foundation and by the Dutch Science Foundation NWO/ FOM We acknowledge helpful comments from D Cohen, F Haake, and R A Jalabert
[1] A Peres, Quantum Theory Concepts and Methods (Kluwer, Dordrecht, 1993)

[2] H M Pastawskı, P R Levstein, G Usaj, J Raya, and J Hirschinger, Physica A 283, 166 (2000)

[3] R A Jalabetl, and H M Pastawskı, Phys Rev Lett 86, 2490 (2001)

[4] F M Cucchietı, H M Pastawskı, and D A Wisnıack1, e-print cond-mat/0102135

[5] A Peres, Phys Rev A 30, 1610 (1984)
[6] E P Wigner, Ann Math 62, 548 (1955), 65, 203 (1957), G Casatı, B V Chirikov, I Guarnerı, and F M Izraılev, Phys Rev E 48, 1613 (1993), V V Flambaum, A A Gribakına, G F Gribakın, and M G Kozlov, Phys Rev A 50267 (1994), $\mathrm{Ph}$ Jacquod and D L Shepelyansky, Phys Rev Lett 75, 3501 (1995)

[7] We assume here that $\Gamma$ is less than the bandwidth $B$ of $H_{0}$ For $\Gamma \geqslant B$ the local spectral density is given by the density of states of $H_{0}$, and accordingly loses 1ts Lorentzian form, cf 
V K B Kota, Phys Rep 347, 223 (2001)

[8] D Cohen and EJ Heller, Phys Rev Lett 84, 2841 (2000), D Cohen, in New Duections in Quantum Chaos, edited by G Casatı, I Guamerı, and U Smılansky (IOS Press, Amsterdam, 2000)

[9] F Haake, Quantum Signatures of Chaos (Sprınger, Berlın, 2000)
[10] G Benettm, L Galganı, and J M Strelcyn, Phys Rev A 14, 2338 (1976)

[11] A Perelomov, Generalized Coherent States and Therr Applcattons (Springer, Berlın, 1986)

[12] F Haake, H Wiedemann, and K Zyczkowk1, Ann Phys (Leipzıg) 1, 531 (1992) 